\title{
Research on the performance evaluation system under the mode of Working Point
}

\author{
Xu Jian-Hua ${ }^{1, a, *}$, Zuo Jia ${ }^{1, b}$ \\ 'Information Technology \& Business Management Department, ChengDu Neusoft University, No.1 \\ Neusoft Road, ChengDu, China \\ axujianhua@nsu.edu.cn, buojia@nsu.edu.cn \\ *corresponding author
}

Keywords: Working Point, Performance evaluation, Human resource management, Data management, Arithmetic.

Abstract. The performance evaluation system, which is based on the mode of Working Point, integrates all kinds of performance appraisal methods in human resource management into a unified platform according to the work management concept. Through the novel Working Point algorithm, systematic data collection, calculation, analysis and management can be realized, and the system can also provide decision-making basis for managers in performance management in accordance with the logic Working Points scoring algorithm, which makes human resource management more scientific, digital, and make the performance evaluation more objective.

\section{Introduction}

Performance evaluation is an important part of performance management. A common performance evaluation includes the Balance, Score-Card, BSC, Key Performance, Indicator, KPI, Management By Objective, MBO, 360 degrees Feedback etc. Those modes have own advantages and disadvantages. Starting from corporate strategies, the BSC method can appraise an enterprise's current and future situations continuously. It satisfies an enterprise's requirements for strategic and long-term development but it is inapplicable to start-up businesses. Emphasizing key indicators that may be quantified in the process of enterprise operation, the KPI method can improve performance appraisal's objectivity and effectiveness but the setting and weight of key indicators are of strong subjectivity. The MBO method decomposes an enterprise's objective to each department and individual. It lays stress on monitoring and enforce-ability. However, it is complicate and ineffective for trans-department job objectives and objectives that cannot be positioned accurately. The 360-degree feedback method appraises performance from multiple dimensions. It can overcome the limitation of single evaluation. 
However, it is mainly applicable to the appraisal for medium and high-level managers. It is inoperative for the appraisal of grass-roots staff. Due to some differences of enterprises in management philosophy, enterprise scales, developing process, operation scope etc., it is very important for companies to choose a performance evaluation mode suiting their needs.

An performance evaluation system based on Working Point mode, it refer to the KPI method and MBO method evaluation, through the analysis of various departments, various positions Working Point, design, calculation, evaluation and feedback, to achieve the enterprise staff performance evaluation.

\section{The design idea of Working Point model}

There is a big difficulty in the process of performance appraisal, i.e. how to evaluate certain employee's effective value accurately? We may use such parameters as education level, age, gender, title, length of service, and work experience to weigh an employee's working ability so as to figure out his/her value to the employer generally. However, these parameters only reflect a static and potential value. Employee's values, attitude toward work, physical condition, thought, and mood will have a big impact on his/her work efficiency. Therefore, it is hard to accurately quantify an employee's actual value to his/her employer.

The basic thought of the Working Point performance appraisal model is to analyze each department and each position's duties in details and design such an position's all Working Point $\mathrm{s}$ and corresponding weight parameter first, then compute and evaluate each position's Working Point through different dimensions and levels, and finally work out such an position's Working Point score, which is treated as a reference for performance appraisal and provided to management departments for human resource management. The setting relationship between the Working Point of Departments and positions will be shown as Figure 1:

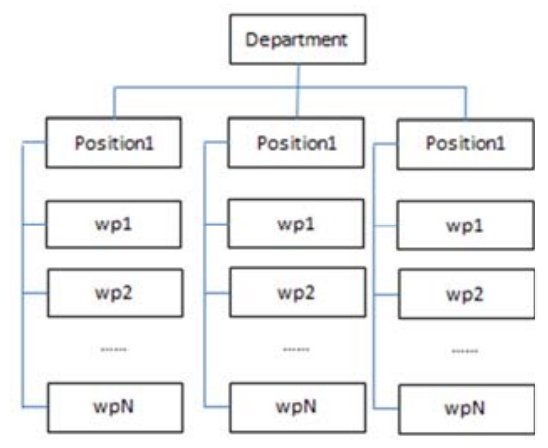

Figure 1: The setting relationship between the Working Point of Departments and positions

Following the process and result-oriented principle, Working Point design describes position duties in details for every position in a department and prepares a position perspective drawing. Based on this, the Working Point corresponding to each performance appraisal is worked out. In order to make the Working Point comply with actualities and remedy possible extreme cases, corresponding weight is set for each Working Point as per degree of completion, importance degree, degrees of association, and 
timeliness. Finally, weighted Working Point score is figured out. Corresponding weight is shown in table 1:

Table 1: The weight set for Working Point

\begin{tabular}{|c|c|c|c|c|}
\hline Working Point & Completeness & Significance & Correlation & Timeliness \\
\hline Reference points & $k 1$ & $k 2$ & $k 3$ & $k 4$ \\
\hline Weight & $w 1$ & $w 2$ & $w 3$ & $w 4$ \\
\hline
\end{tabular}

Notes: The range of the value of $K$ is $0-10$; the value range of $W$ is $0^{-1}$

Its computational formula of Working Point is shown as below:

$$
w p=\sum_{i=1}^{4}\left(k_{i} w_{i}\right)
$$

When computing a Working Point score, a weight may be set for each Working Point according to actualities and statistical computation will be done on a weighted basis. Its computational formula is shown as below:

$$
W P=\sum_{i=1}^{n}\left((w p)_{i} W_{i}\right)
$$

Notes: Each Working Point weight W range of 0-1

In nature, a performance evaluation system based on Working Point mode is an objective and standard appraisal method, which figures out corresponding performance appraisal results by comparing a department or staff's actual work performance with preset job objectives. The system supports an appraisal of three dimensions, i.e. objective self-appraisal, direct superior's appraisal, and competent leadership's appraisal. All Working Point parameters may be preset and changed. At the time of appraisal, an operator may carry out automatic computation and statistics by entering numbers and offer appraisal results to managers for decision making.

\section{Design of performance appraisal management system based on Working Point}

\subsection{System architecture design}

With classic Linux + Apache + MySQL + PHP (LAMP) as a development architecture, the system uses PHP + MySQL technology as a development tool and adopts B/S structure to show the system's operation. Meanwhile, it supports App mobile applications developed based on Andriod. Thus uses can use it easily, development costs are reduced, and working efficiency is increased.

The system's framework is made up of two parts:

Staff Working Point management subsystem: used for input, setting, appraisal, and feedback of staff 's Working Point, complete quantitative human resource appraisal in the form of daily report, and based on this, figure out staff's Working Point score of monthly work, and work out corresponding data reports for analysis and decision making. 
Department Working Point management subsystem: used to carry out administrative quantitative appraisal to a department's Working Point on a monthly, quarterly, and annual basis and work out corresponding data reports for analysis and decision making. Software function module of system is shown in figure 2:

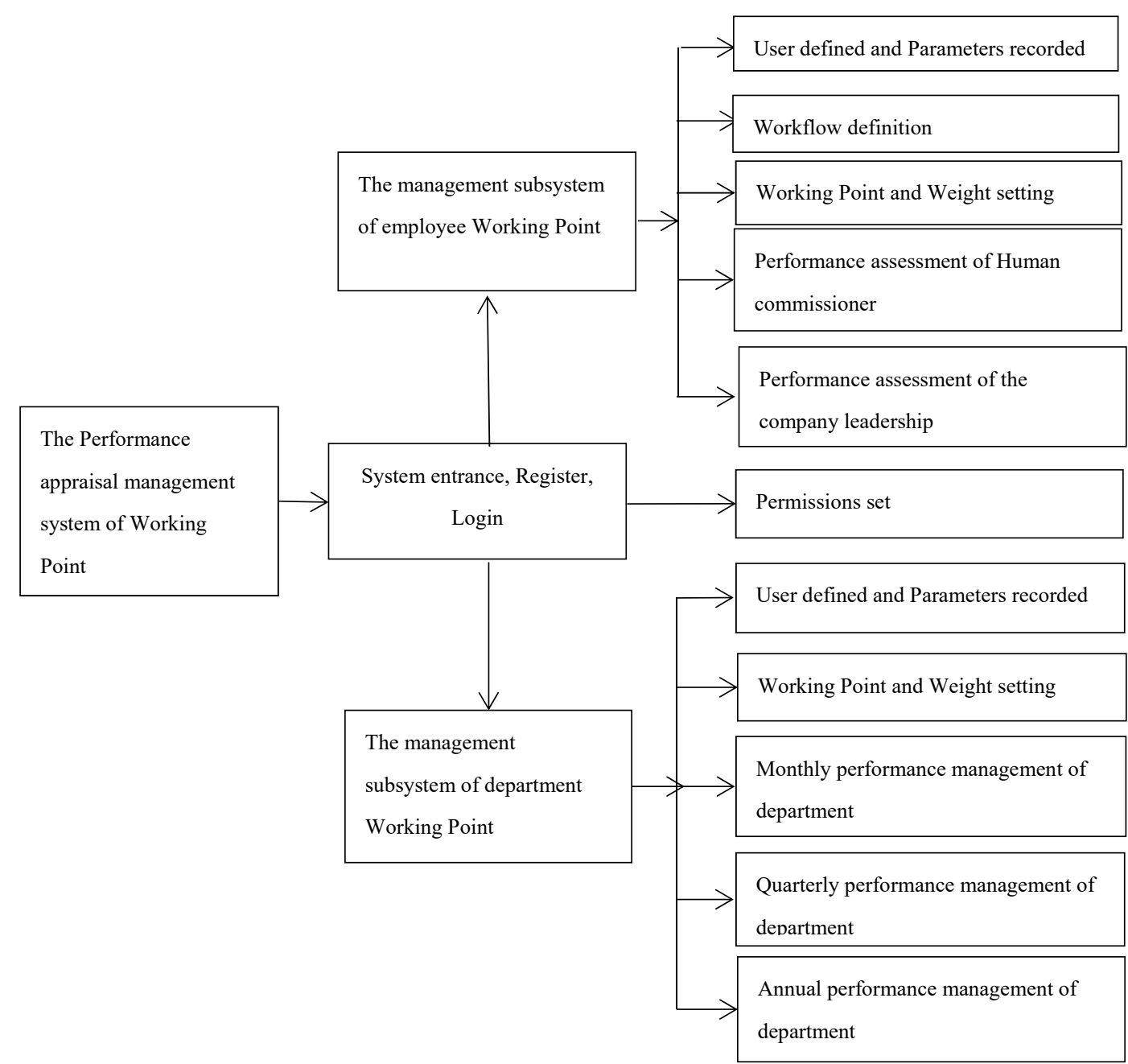

Figure 2: software function module of WP performance appraisal management systems

\subsection{Software function module description}

\subsubsection{Working Point performance evaluation system portal design}

The system is divided into four modules: user registration, user login, performance evaluation subsystem entrance, and data analysis and performance evaluation (as shown in Figure 3) 


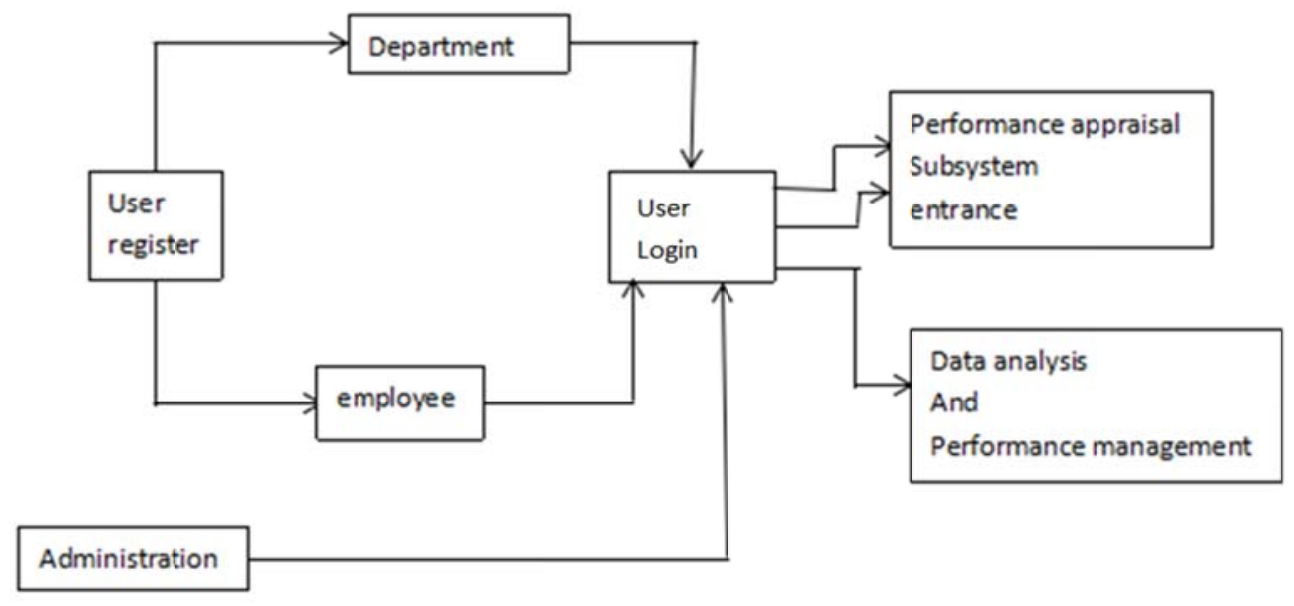

Figure 3 Portal function module of Working Point system

The staff and the Department that first use the system need to register information, so that the system can input the original data. The staff needs to fill in the registration information including the number, name, gender, year of entry, department, position and other information. The Department needs to fill in the information including Department number, Department name, Departmental responsibilities, Department architecture and other information.

There are three kinds of user identity, namely, employee, department and administrator. For employees and departments, after a successful login, it will jump to the performance evaluation subsystem module. While for administrators, it will jump to the data analysis and performance management module.

Performance evaluation subsystem provides the appropriate permissions for all departments and individual performance evaluation, including work input, value evaluation/self-rating score, calculation and statistical functions. Employees and departments can login to boot into the corresponding module according to the system operation.

\subsubsection{Data analysis and performance management subsystem}

An administrator or authorized managerial staff may log in the module to search and analyze corresponding subsystem's performance appraisal details, obtain corresponding performance appraisal data, which are shown in different charts, based on customized search conditions, and print them online or export them in EXCEL format.

\subsubsection{Mobile terminal application system}

In order to adapt to a more flexible way and improve work efficiency, for the performance evaluation system and Working Point, an app mobile application module has been also developed based on Andriod, to provide query and analysis function for the enterprise managers. Working principle: the Android client will send the POST request or GET request to the specified PHP file 
through the class "httpurlconnection", the PHP server accepts parameters from the client and then returns JSON data back to the client, and then the mobile terminal screen shows the corresponding format as required by the client.

\subsection{System database design}

The system's database software is MySQL Community Server5.5.19, with a secondary structure. It is divided into raw database and target database. Data that are sent from each subsystem to database server are written in raw database first and then written in target database according each data's attribute. System software's data operation points only to target database and does not contact raw database. In this way, data security is guaranteed to the largest extent. Target database includes data dictionary, employee information table, department information table, administrator (leader) information table, Working Point weight parameter design table, Working Point appraisal statistical table, and system log sheet, etc. Data analysis method: by completing a series of stored procedure, corresponding performance appraisal data are obtained from data sheet and a HTML page is generated automatically to go back to client terminal. The stored procedure's name is made up of search condition type name and statistical type name, e.g. storage process GetData_Emp_SubSys_Dep_Gra_ByChk. Statistical data may be searched by parameter appraisal subsystem, department, and job Working Point, etc. The system will list out appraisal records of the department/employee searched.

\subsection{System safety design}

\subsubsection{Permission design}

According to the actual needs of the business, the users are defined into three levels, i.e. Department, employee and administrator. The users of different roles can access to the system through different log in interfaces. At the same time, the implementation of management of authorized users prevents user operation beyond the specified scope.

\subsubsection{Data security}

Important data, e.g. account number, password, database IP, are encrypted by using such methods as RSA and DES. For Database security, image management has been used for the database. User identification and data access control are done for database access. Standard backup software is used to realize online data storage and backup. Tape backup medium is used to guarantee data security.

\section{Conclusion}

Performance appraisal is a core part of performance management. The accuracy of appraisal determines the correctness of incentive control, assessment and feedback. The Working Point model-based performance appraisal system reflects a design concept of double appraisal to course of 
work and work results. Meanwhile, by setting various weight parameters, it has higher application elasticity and can better satisfy actual demands in corporate human resource management. This system has shown a very good practical value through over two year's practice.

\section{References}

[1]S Wahab, EAA Malek. Human Resource Management Information System and Job Performance. (2014) Environmental Pollution, 20, 143-166.

[2]Yan, aiming, Li, linghong. Exploratory Research on Difficulty Coefficient Application in Performance Evaluation Field. (2014) Science and Technology Management Research, 34(7), 49-51.

[3]Li, ping. Information management system based on financial industry performance management. (2015) Modern Electronics Technique, 1, 112-114.

[4]Chen, peiran. Dynamic spiral model of employee performance evaluation index system. (2015) Enterprise management, 8, 108-110.

[5]Xu, yaning. Performance evaluation of enterprise middle level managers based on uncertain information. (2016) Statistics and decision, 12, 177-179. 